\title{
Acoustic pulse reflectometry in musical wind instrument research
}

\author{
D.M. CAMPBELL, R. PARKS and D. SHARP \\ Dept. of Physics, J.C.M.B., Kings Buildings, University of Edinburgh, Edinburgh, U.K.
}

\begin{abstract}
Criteria for the design of an acoustic pulse reflectometer specifically intended for musical instrument research are discussed. Different types of pulse source are considered, and various approaches to the signal processing of the reflected pulse are evaluated., Finally, the practical utility of the technique for instrument designers and manufacturers is discussed.
\end{abstract}

\section{Introduction}

The technique of acoustic pulse reflectometry was originally developed for seismological studies [1]. In recent years it has been applied to the investigation of ducts of varying cross-section, both in the medical area [2] and in the field of musical instrument research [3]. Although encouraging results have been achieved in both of these areas, the precision of the technique has been limited by two factors. Firstly, it has proved difficult to devise an experimental arrangement in which an acoustic pulse with the ideal spectral content is cleanly injected into and recovered from the test object, with the high reproducibility necessary for signal averaging. Secondly, the computational techniques used to analyse the reflected signal have been relatively unsophisticated.

This paper discusses the criteria for the design of an acoustic pulse reflectometer specifically intended for musical instrument research. Types of pulse source and approaches to signal processing are evaluated. Finally, the practical utility of the technique for instrument designers and makers is discussed. 


\section{Basis of the technique}

In the acoustical study of wind instruments, two types of measurement have proved of particular value. One is direct measurement of the bore profile; the other is the evaluation of input impedance, defined as the ratio of acoustic pressure to volume air flow rate at the entrance to the instrument. These two aspects are closely related, since in principle one can be calculated from the other. In practice, however, complications from such features as side holes limit the accuracy with which the impedance can be calculated from the bore profile. In addition, it is not always possible to obtain access to the complete bore of an instrument with accurate measuring implements. Direct measurement of input impedance has thus become an important technique.

A major problem with the conventional approach to the experimental study of acoustic impedance has been the necessity for a measurement of the volume flow rate, a difficult technique under the conditions applicable to musical wind instruments. The great advantage offered by acoustic pulse reflectometry is that it requires only pressure measurement, yet it can yield absolute values of both input impedance and bore profile.

The technique involves the injection of a sound pressure pulse into the instrument, and the recording of the sound reflected back from the instrument. This reflected sound consists of a primary reflection from each impedance change in the instrument, plus a series of multiple reflections from within the instrument. Deconvolution of the reflected signal with the incident pulse shape yields the input impulse response of the instrument. Further computation, based on an inversion program such as that of Ware and Aki [1] gives the input impedance and the bore reconstruction.

\section{Physical constraints on the apparatus}

Fig. 1 is a schematic diagram of an acoustic pulse reflectometer of the type currently in use in Edinburgh. A horn loudspeaker driver receives an electrical pulse from a pulse generator and audio amplifier. The driver is coupled to a coiled copper tube. At a distance $l_{1}$ along the tube, a microphone records the passage of the resulting input pressure pulse, of length $\tau$, and also the returning wave which has been reflected from an object coupled to the far end of the tube, a distance $\mathrm{I}_{2}$ from the microphone.

The length $l_{1}$ (source to microphone) is determined by the fact that a secondary reflection from the source arrives at the microphone a time $t_{s}=2 l_{1} / c$ after the passage of the first reflected wave. To avoid confusion it is desirable that all the reflections from the instrument should be collected within this time. If the instrument has a length $\mathrm{x}$, the interval between first reflections from entrance and exit is $\Delta t=2 x / c$. However, there may be $n$ multiple reflections before the signal 
falls below the noise level. In that case the total time span of the reflected signal is $\Delta \mathrm{t}=2 \mathrm{nx} / \mathrm{c}$, and a complete separation would require $\mathrm{l}_{1}<\mathrm{nx}$. The present reflectometer has $l_{1}=3 \mathrm{~m}$, so that, if multiple reflections up to order three are significant, the maximum length of instruments which can be studied is $x=1 \mathrm{~m}$.

The length $\mathrm{l}_{2}$ (microphone to instrument input) is determined by the requirement that the input and reflected signals must be separated in time. The first reflection reaches the microphone a time $t_{f}$ after the passage of the leading edge of the input pulse, where $t_{f}=2 l_{2} / c$. Thus $\tau<t_{f}$, and $l_{2}>\tau c / 2$. In the present apparatus, $1_{2}=3 \mathrm{~m}$, allowing pulses up to $15 \mathrm{~ms}$ long to be used.

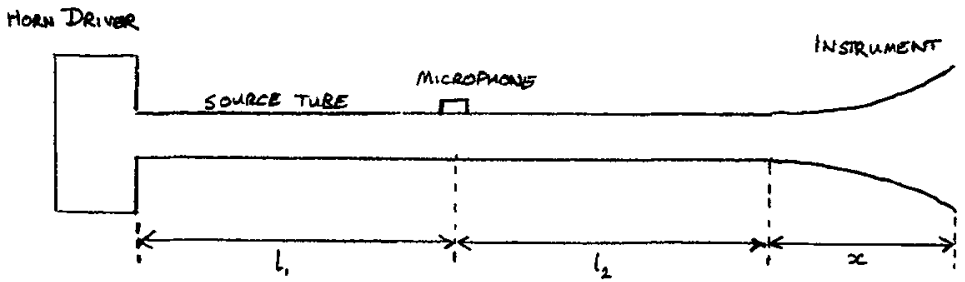

Figure 1

\section{Pulse generation}

Fig. 2 shows a typical input pulse generated by the present apparatus, while Fig. 3 shows its Fourier spectrum. In principle, the ideal input pulse would be a delta function, since this would have a uniform Fourier spectrum and would give the input impulse response without deconvolution. Marshall [4] has implemented a technique, using a computer generated pulse, which created a close approximation to an impulse pressure wave, thus permitting $l_{2}$ to be reduced to a few centimetres. This has the advantage of reducing attenuation in the source tube. A disadvantage of very short input pulses is that they require high amplitude in order to give adequate power.

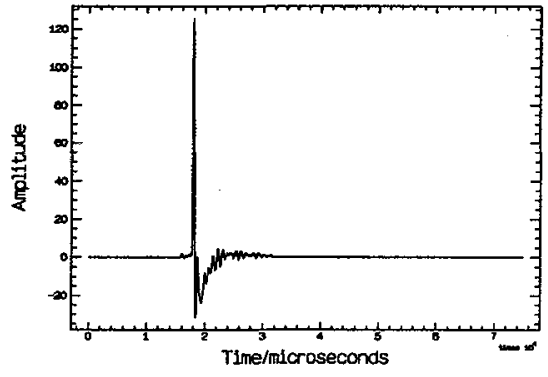

Figure 2

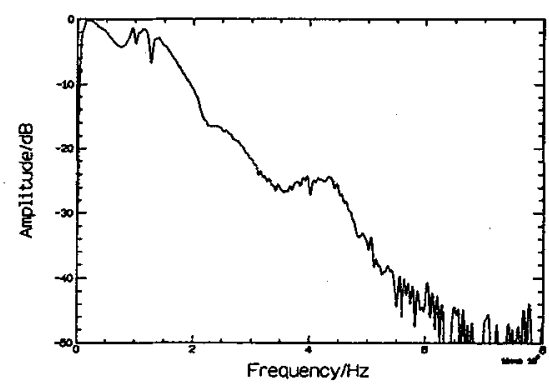

Figure 3 


\section{Computational techniques}

The algorithm of Ware and Aki [1] has become established as the standard method for computing input impedance. A simpler method, involving the recording only of first reflections, gives reasonable results much more quickly for reconstruction. Figures 4 and 5 show bore reconstructions for a stepped tube and a trumpet bell, using this approach. Its speed allows for real-time display of changing bore profiles [4] (for example, the opening and closing of a trumpet valve while playing). Normally, however, the greater accuracy of the Ware-Aki approach is preferable.

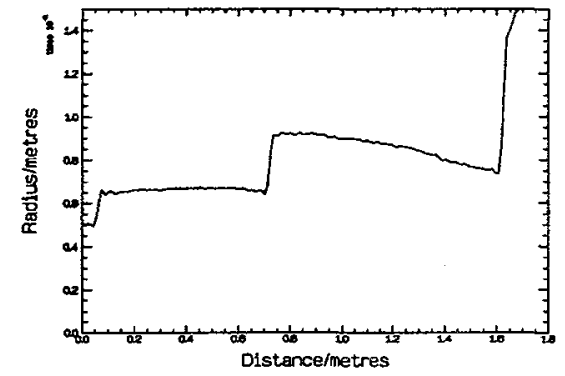

Figure 4

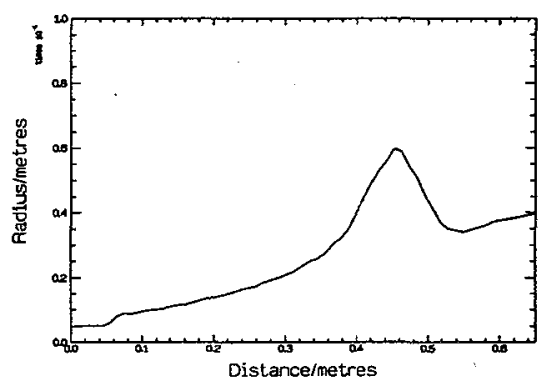

Figure 5

\section{Potential of pulse reflectometry}

With optimisation of the pulse input and the analysis technique, pulse reflectometry promises to become a more precise and useful tool in the study of the behaviour of wind instruments. The two-microphone technique of Louis et al [5] and the source reflection calibration method of Marshall [6] offer the possiblity of much more compact reflectometers.

\section{References}

1 Ware,J.A. and Aki,K. Continuous and discrete inverse scattering problems in a stratified elastic medium. I: Planes at normal incidence. JASA(1969) 54(4),911-921.

2 Jackson,A.C., Butler,J.P., Millet,E.J., Hoppin,F.G. and Dawson,S.V. Airway geometry by analysis of acoustic pulse response measurements. J.Appl.Physiol(1977) 43(3),523-536.

3 Watson,A.P. and Bowsher,J.M. Impulse measurements on brass musical instruments. Acustica(1988) 66,171-174.

4 Marshall,I. PhD thesis

5 Louis,B., Glass,G., Krasen,B. and Fredberg,J. Airway area by acoustic reflection. J.Boimed.Eng. in press(1993)

6 Marshall,I. Acoustic reflectometry with an arbitrarily short source tube. JASA(1992) 91(6),3558-3563. 